\title{
The AF protocol of cooperative communication system
}

\author{
Lina Yuan ${ }^{1, a}$, Jing Gong ${ }^{1, b}$, Huajun Chen ${ }^{1, c}$, Yiwang Huang ${ }^{1, d}$ \\ ${ }^{1}$ College of Information Engineering, Shenzhen University, Guangdong, 518060, P.R.China. \\ a893422817@qq.com, b996075565@qq.com, ${ }^{\text {c3 } 390832683 @ q q . c o m, ~}{ }^{\text {d4 } 458051500 @ q q . c o m}$
}

Keywords: cooperative communication system, cooperative relay technology, AF and DF protocols,

\begin{abstract}
Wireless communication system using cooperative transmission technology, at the receiving node by combining data samples from multiple independent fading channel, can effectively resist the influence of channel fading, obtain diversity gain, and improve the transmission reliability of the system. The purpose of cooperative communication technology is to solve the problem of communication effectiveness. This article expounds the amplify-and-forward(AF) protocol of the cooperative relay technology that is one of the key technology in cooperative communication technology, and discusses its ergodic capacity(EC). Theory proves that two-way relay can greatly improve the rate of information transmission
\end{abstract}

\section{Introduction}

Cooperative communication technology is one of the most important research focus in the field of wireless communication nowadays. When to design a wireless communication system, it needs to consider overcoming the characteristics of wireless channel fading, expanding channel capacity and improving the channel quality, while cooperative relay technology can overcome the above problems[1]. Cooperative relay technology can greatly improve the quality of communication, resist the influence of wireless channel fading, shadow effect and multi-path effect, expand the scope of communication, and reduce the mobile terminals, etc.Cooperative relay technology can greatly improve the quality of communication, resist the influence of wireless channel fading, shadow effect and multi-path effect, expand the scope of communication, and reduce the mobile terminals, etc. However, due to the limitation of practical half-duplex relay communication system, the relay technology also brings the loss of spectrum efficiency at the same time in improving the performance of wireless communication. In this case, two-way relay arises at the historic moment. Two-way relay can receive and forward signals of two end users to greatly improve the rate of information throughput(RIT).

The rest of this paper is organized as follows. Section 2 expounds the principle of two-way relay system, analyzes in detail two relay protocols-AF protocol. Section 3 analyzes in detail the AF protocol of two relay protocol and deduces its ergodic capacity(EC) under the condition of Rayleigh fading channel for two-way relay system. Section 4 concludes the paper and proposes future work.

\section{The protocol of two-way relay system}

Considering a two-way relay communication system, the user nodes-A and $\mathrm{B}$ exchange information each other. But because there is no direct link between A and B, and needs to forward information through the relay node-R, as shown in Fig. 1[3]. Supposing the system goes through flat fading, and adopts time-division duplex mode, then the channels are reciprocity. Take the system as shown in Fig. 1 as an example to discuss the different protocols two-way relay system. 


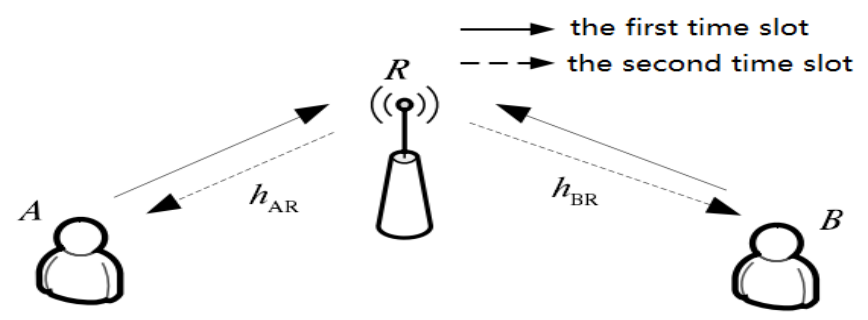

Fig.1 The model of two-way relay system

In the first time slot, A and B simultaneously send their own signals to R. In this way, the received signals at $\mathrm{R}$ can be expressed as (1).

$$
y_{R}=h_{A R} x_{A}+h_{B R} x_{B}+n_{R}
$$

Among it, $x_{A}$ and $x_{B}$ is the sent signal of $\mathrm{A}$ and $\mathrm{B}$ respectively. $h_{A R}$ is the fading channel coefficient between $\mathrm{A}$ and $\mathrm{R}, h_{B R}$ is the fading channel coefficient between $\mathrm{B}$ and $\mathrm{R}$, and $n_{R}$ is AWGN with the power- $\sigma^{2}$ at the R.

In the second time slot, $\mathrm{R}$ firstly processes the received signals, and then transmits information to $\mathrm{A}$ and $\mathrm{B}$. Therefore $\mathrm{R}$ amplifies the received signals and then forwards them, while during the amplification process, the following amplification factor is used:

$$
\alpha=\sqrt{\frac{P_{R}}{P_{A}\left|h_{A R}\right|^{2}+P_{B}\left|h_{B R}\right|^{2}+\sigma^{2}}}
$$

Among it, $P_{R}, P_{A}$ and $P_{B}$ present the sent power of $\mathrm{R}, \mathrm{A}$ and $\mathrm{B}$ respectively. Thus the received signals of $\mathrm{A}$ and $\mathrm{B}$ can be expressed in the following formulations separately.

$$
\begin{aligned}
& y_{A}=h_{A R} \alpha y_{R}+n_{A}=h_{A R} \alpha\left(h_{A R} x_{A}+h_{B R} x_{B}+n_{R}\right)+n_{A} \\
& y_{B}=h_{B R} \alpha y_{R}+n_{B}=h_{B R} \alpha\left(h_{A R} x_{A}+h_{B R} x_{B}+n_{R}\right)+n_{B}
\end{aligned}
$$

Among them, $n_{A}$ and $n_{B}$ are AWGN with the variance- $\sigma^{2}$ at A and B. Because A and B are both known their own sent signals, their own self-interference of the formulations-(3) and (4) can be completely eliminated[3]. And the instantaneous received SNR of two one-way channel- $A \rightarrow R \rightarrow B$ and $B \rightarrow R \rightarrow A$ in this two-way relay communication system can be obtained respectively as:

$$
\begin{gathered}
\Gamma_{B}^{A F}=\frac{\left|h_{A R}\right|^{2}\left|h_{B R}\right|^{2} \gamma_{A} \gamma_{R}}{\gamma_{A}\left|h_{A R}\right|^{2}+\left(\gamma_{R}+\gamma_{B}\right)\left|h_{B R}\right|^{2}+1} \\
\Gamma_{A}^{A F}=\frac{\left|h_{A R}\right|^{2}\left|h_{B R}\right|^{2} \gamma_{B} \gamma_{R}}{\left(\gamma_{A}+\gamma_{R}\right)\left|h_{A R}\right|^{2}+\gamma_{B}\left|h_{B R}\right|^{2}+1}
\end{gathered}
$$

Among it, $\gamma_{A} \stackrel{\Delta}{=} P_{A} / \sigma^{2}, \stackrel{\Delta}{\gamma_{B}} P_{B} / \sigma^{2}$, and $\gamma_{R} \stackrel{\Delta}{=} P_{R} / \sigma^{2}$. Therefore, the instantaneous information rate of two one-way channel- $A \rightarrow R \rightarrow B$ and $B \rightarrow R \rightarrow A$ can be expressed respectively as

$$
\begin{gathered}
I_{B}^{A F}=\frac{1}{2} \log _{2}\left(1+\Gamma_{B}^{A F}\right) \\
I_{A}^{A F}=\frac{1}{2} \log _{2}\left(1+\Gamma_{A}^{A F}\right)
\end{gathered}
$$

Note that to estimate the signal-to-noise ratio of two-way relay systems, there still needs to further estimate noise power of nodes. In the actual relay system, it can be sent pilot information to estimate the noise power. Under the condition of known pilot information and channel state information, use the received pilot signals to estimate the noise power[4-5]. 


\section{An analysis of two-way AF relay system for its ergodic capacity(EC)}

The EC of two-way AF relay system[7,10] is the sum of two one-way channel for their EC, which can be expressed as:

$$
C_{T}^{A F} \stackrel{\Delta}{=} C_{A}+C_{B}
$$

Among it, $C_{A} \stackrel{\Delta}{=} \mathrm{E}_{h_{A R}, h_{B R}}\left(I_{A}^{A F}\right)$ and $C_{B} \stackrel{\Delta}{=} \mathrm{E}_{h_{A R}, h_{B R}}\left(I_{B}^{A F}\right)$, respectively expressed as:

$$
\begin{aligned}
& C_{A}=\mathrm{E}_{h_{A R}, h_{B R}}\left[\frac{1}{2} \log _{2}\left(1+\Gamma_{A}^{A F}\right)\right]=\mathrm{E}_{h_{A R}, h_{B R}}\left[\frac{1}{2} \log _{2}\left(1+\frac{\gamma_{B} \gamma_{R}\left|h_{A R}\right|^{2}\left|h_{B R}\right|^{2}}{\left(\gamma_{A}+\gamma_{R}\right)\left|h_{A R}\right|^{2}+\gamma_{B}\left|h_{B R}\right|^{2}+1}\right)\right] \\
& C_{B}=\mathrm{E}_{h_{A R}, h_{B R}}\left[\frac{1}{2} \log _{2}\left(1+\Gamma_{B}^{A F}\right)\right]=\mathrm{E}_{h_{A R}, h_{B R}}\left[\frac{1}{2} \log _{2}\left(1+\frac{\gamma_{A} \gamma_{R}\left|h_{A R}\right|^{2}\left|h_{B R}\right|^{2}}{\gamma_{A}\left|h_{A R}\right|^{2}+\left(\gamma_{B}+\gamma_{R}\right)\left|h_{B R}\right|^{2}+1}\right)\right]
\end{aligned}
$$

While the exact resolution calculation of $C_{A}$ and $C_{B}$ is very complex, at present there is no literature to give exact solution. This section will give the upper bound of $C_{A}$ and $C_{B}$. According to the in-equation- $x y /(x+y+1) \leq \min (x, y)$, the upper bound of $C_{A}$ can be expressed as:

$$
\begin{aligned}
& C_{A}=\mathrm{E}_{h_{A R}, h_{B R}}\left[\frac{1}{2} \log _{2}\left(1+\frac{\gamma_{R}}{\gamma_{A}+\gamma_{R}} \frac{\left(\gamma_{A}+\gamma_{R}\right)\left|h_{A R}\right|^{2}\left|h_{B R}\right|^{2} \gamma_{B}}{\left(\gamma_{A}\right)\left|h_{A R}\right|^{2}+\left|h_{B R}\right|^{2} \gamma_{B}+1}\right)\right] \\
& \leq \mathrm{E}_{h_{A R}, h_{B R}}\left[\frac{1}{2} \log _{2}\left(1+\frac{\gamma_{R}}{\gamma_{A}+\gamma_{R}} \min \left(\left|h_{A R}\right|^{2}\left(\gamma_{A}+\gamma_{R}\right),\left|h_{B R}\right|^{2} \gamma_{B}\right)\right)\right] \stackrel{\Delta}{=} C_{A}^{\text {upper }}
\end{aligned}
$$

According to the assumption: $\left|h_{A R}\right|^{2},\left|h_{B R}\right|^{2}$ separately subject to the exponential distribution of $\Omega_{A R}, \Omega_{B R}$, so the PDF of $Y \stackrel{\Delta}{=} \min \left(\left|h_{A R}\right|^{2}\left(r_{A}+r_{R}\right),\left|h_{B R}\right|^{2} r_{R}\right)$ can be expressed as:

$$
f_{Y}(y)=\left(\frac{1}{\left(\gamma_{A}+\gamma_{R}\right) \Omega_{A R}}+\frac{1}{\gamma_{R} \Omega_{B R}}\right) \exp \left[-\left(\frac{1}{\left(\gamma_{A}+\gamma_{R}\right) \Omega_{A R}}+\frac{1}{\gamma_{R} \Omega_{B R}}\right) y\right]
$$

So $C_{A}^{\text {upper }}$ can be expressed as:

$$
\begin{aligned}
& C_{A}^{\text {upper }}=\frac{1}{2}\left(\frac{1}{\left(\gamma_{A}+\gamma_{R}\right) \Omega_{A R}}+\frac{1}{\gamma_{B} \Omega_{B R}}\right) \times \int_{0}^{\infty} \log _{2}\left(1+\frac{\gamma_{R} y}{\gamma_{A}+\gamma_{R}}\right) \exp \left[-\left(\frac{1}{\left(\gamma_{A}+\gamma_{R}\right)}+\frac{1}{\gamma_{B} \Omega_{B R}}\right) y\right] d y \\
& =\frac{1}{2 \ln 2} \exp \left(\frac{1}{\gamma_{R} \Omega_{A R}}+\frac{\gamma_{A}+\gamma_{R}}{\gamma_{B} \gamma_{R} \Omega_{B R}}\right) E_{1}\left(\frac{1}{\gamma_{R} \Omega_{A R}}+\frac{\gamma_{A}+\gamma_{R}}{\gamma_{B} \gamma_{R} \Omega_{B R}}\right)
\end{aligned}
$$

According to the in-equation- $x y /(x+y+1) \leq \min (x, y)$, the upper bound of $C_{B}$ can be expressed as:

$$
C_{B} \leq \mathrm{E}_{h_{A R}, h_{B R}}\left[\frac{1}{2} \log _{2}\left(1+\frac{\gamma_{R}}{\gamma_{B}+\gamma_{R}} \min \left(\left|h_{A R}\right|^{2} \gamma_{A},\left|h_{B R}\right|^{2}\left(\gamma_{B}+\gamma_{R}\right)\right)\right)\right] \stackrel{\Delta}{=} C_{B}^{\text {upper }}
$$

Let $Y \stackrel{\Delta}{=} \min \left(\left|h_{A R}\right|^{2} r_{A},\left|h_{B R}\right|^{2}\left(r_{B}+r_{R}\right)\right)$, so the PDF of $Y$ can be expressed as:

$$
f_{Y}(y)=\left(\frac{1}{\left(\gamma_{B}+\gamma_{R}\right) \Omega_{B R}}+\frac{1}{\gamma_{A} \Omega_{A R}}\right) \exp \left[-\left(\frac{1}{\left(\gamma_{B}+\gamma_{R}\right) \Omega_{B R}}+\frac{1}{\gamma_{A} \Omega_{A R}}\right) y\right]
$$

And $C_{B}^{\text {upper }}$ can be expressed as: 


$$
\begin{aligned}
& C_{B}^{\text {upper }}=\frac{1}{2}\left(\frac{1}{\left(\gamma_{B}+\gamma_{R}\right) \Omega_{B R}}+\frac{1}{\gamma_{A} \Omega_{A R}}\right) \times \int_{0}^{\infty} \log _{2}\left(1+\frac{\gamma_{R} y}{\gamma_{B}+\gamma_{R}}\right) \exp \left[-\left(\frac{1}{\left(\gamma_{B}+\gamma_{R}\right)}+\frac{1}{\gamma_{A} \Omega_{A R}}\right) y\right] d y \\
& =\frac{1}{2 \ln 2} \exp \left(\frac{1}{\gamma_{R} \Omega_{B R}}+\frac{\gamma_{B}+\gamma_{R}}{\gamma_{A} \gamma_{R} \Omega_{A R}}\right) E_{1}\left(\frac{1}{\gamma_{R} \Omega_{B R}}+\frac{\gamma_{B}+\gamma_{R}}{\gamma_{A} \gamma_{R} \Omega_{A R}}\right) \\
& C^{\text {upper }}=\frac{1}{2 \ln 2}\left\{\exp \left(\frac{1}{\gamma_{R} \Omega_{A R}}+\frac{\gamma_{A}+\gamma_{R}}{\gamma_{B} \gamma_{R} \Omega_{B R}}\right) E_{1}\left(\frac{1}{\gamma_{R} \Omega_{A R}}+\frac{\gamma_{A}+\gamma_{R}}{\gamma_{B} \gamma_{R} \Omega_{B R}}\right)\right. \\
& \left.+\exp \left(\frac{1}{\gamma_{R} \Omega_{B R}}+\frac{\gamma_{B}+\gamma_{R}}{\gamma_{A} \gamma_{R} \Omega_{A R}}\right) E_{1}\left(\frac{1}{\gamma_{R} \Omega_{B R}}+\frac{\gamma_{B}+\gamma_{R}}{\gamma_{A} \gamma_{R} \Omega_{A R}}\right)\right\}
\end{aligned}
$$

\section{Conclusion and future work}

This paper mainly gives a detailed analysis of one kind of relay protocol, that is, amplify and forward(AF) protocol, and derives their ergodic capacity(EC) under the condition of Rayleigh fading channel. Our future work will compare the EC of AF protocol with DF protocol by simulation and seek whose implementation complexity is low and which is more suitable for application in actual two-way relay system. When to send information, and how to select the relay node, keep synchronization and assign the power is further worth researching.

\section{ACKNOWLEDGEMENTS}

This work was supported by the Collaborative Fund Project of Science and Technology Agency in Guizhou Province Marked by the word LH on 7487[2014] and 7476[2014], partly supported by the National Natural Science Foundation of China(NO.61562703), and the project of education and cooperation for talent team word in Guizhou in 2015(NO:[2015]67).

\section{References}

[1] Hatem Boujemâa.Static hybrid amplify and forward (AF) and decode and forward (DF) Relaying for cooperative systems.Physical Communication 4, pp.196-205,2011.

[2] J. N. Laneman, D. N. Tse, and G. W. Wornell,"Cooperative diversity in wireless networks: Efficient protocols and outage behavior," IEEE Transactions on Information Theory, vol. 50, no. 12, pp. 3062-3080,December 2004.

[3] B. Rankov and A. Wittneben, "Spectral efficient protocols for half-duplex fading relay channels," IEEE J. Sel. Areas Commun., vol. 25, no. 2, pp. 379-389, Feb 2007.

[4] Meulen, E.: 'Three-terminal communication channels', Adv. Appl. Probab., vol.3,no.1,pp.120-154,1973.

[5] Yong Soo Cho, Jaekwon Kim, Won Young Yang, Chung-Gu Kang. MIMO-OFDM Wireless Communications with MATLAB[M].IEE E PRESS, John Wiley \& Sons (Asia) Pte Ltd. Pp. $71-75,2010$.

[6] J. N. Laneman and G. W. Wornell, "Energy efficient antenna sharing and relaying for wireless networks," in Proc. IEEE WCNC, pp.7-12, Oct. 2000.

[7] I. S. Gradshteyn and I. M. Ryzhik, Table of Integrals, Series, and Products, 7th edition. San Diego, CA, USA: Academic Press, 2007.

[8] L. Fan, X. Lei, and W. Li, "Exact Closed-Form Expression for Ergodic Capacity of Amplifyand-Forward Relaying in Channel-Noise-Assisted Cooperative Networks with Relay Selection," IEEE Commun. Lett., vol. 15, no. 3, pp.332-333, Mar 2011. 
[9] T. M. Cover and A. A. EL Gamal, "Capacity theorems for the relay channel," IEEE Trans. Info. Theory, vol. 25, no. 5, pp. 572-584, Sep 1979.

[10]Muhaidat, H., Uysal, M.: 'Cooperative diversity with multiple-antenna nodes in fading relay channels', IEEE Trans. Wirel. Commun., 7, (8), pp.3036-3046, 2008.

[11]L. Zhang, C.-C. Shen, L. Cimini, and B. Gui, "Networking with cooperative communications: Holistic design and realistic evaluation," IEEE Commun. Mag., vol. 47, no. 8, pp.113-119,Aug 2009.

[12]Cheng, W., Ghogho, M., Huang, Q., Ma, D., Wei, J.: 'Cooperative beamforming for OFDM-based amplify-and-forward relay networks’, Phys. Commun., pp.305-312, April 2011. 